\title{
Bidirectional control of saccadic eye movements by the disconnected cerebral hemispheres
}

\author{
H.C. Hughes ${ }^{1,2}$, P.A. Reuter-Lorenz ${ }^{1,2 *}$, R. Fendrich ${ }^{2 * *}$, and M.S. Gazzaniga ${ }^{2 * *}$ \\ ${ }^{1}$ Department of Psychology, Dartmouth College and ${ }^{2}$ Program in Cognitive Neuroscience, \\ Dartmouth Medical School, Hanover, NH 03755, USA
}

Received October 25, 1991 / Accepted April 22, 1992

\begin{abstract}
Summary. The present investigation demonstrates that callosotomy patient J.W. can generate either leftward or rightward saccades in response to color cues presented unilaterally. When asked to name the colors, performance was at chance for left visual field presentations, demonstrating a disability in interhemispheric transfer of chromatic information. The successful control of saccadic direction based on discriminative color cues that appear confined to a single hemisphere may suggest a capacity for bidirectional control of saccadic eye movements in the disconnected cerebral hemispheres.
\end{abstract}

Key words: Saccadic eye movements - Corpus callosum Cerebral hemispheres - Ipsilateral control

\section{Introduction}

Electrical stimulation (e.g. Robinson 1972; Robinson and Fuchs 1969) and recording studies (e.g. Bizzi and Schiller 1970; Bruce and Goldberg 1984; Sparks and Pollack 1977; Wurtz and Albano 1980) reveal that cortical and collicular components of the saccadic system are organized to direct saccades contralaterally. In virtually all systems studied thus far, including the superior colliculus [SC], and frontal eye fields [FEF], electrical microstimulation serves to elicit saccades that are directed into the contralateral visual field. Recordings of presaccadic activity of single neurons corroborate the general finding of contralateral control. The lone exception appears to be the supplementary eye fields (e.g., Schlag and Schlag-Rey 1987; Schall 1991a, b), where ipsilaterally directed saccades are occasionally elicited.

Contralateral saccadic control is compatible with the contralateral projection of each visual hemifield onto the cerebral hemispheres and superior colliculus. It is curious

\footnotetext{
* Present addresses: Dept. of Psychology, Univ. of Michigan, USA

** Center for Neurobiology, Univ. of Calif., Davis, USA

Correspondence to: H.C. Hughes
}

then to find suggestions that the cerebral hemispheres of humans can direct saccades into either the contralateral or the ipsilateral visual fields. These suggestions derive from observations on hemispherectomy patients and callosotomy patients. Reports indicate that human hemispherectomy patients can generate saccades into their blind hemifield (Sharpe et al. 1979; Troost et al. 1972). In some conditions, these saccades were apparently visually elicited whereas in others the patients could generate saccades directed contralaterally to the excised hemisphere on request. While saccades elicited by visual stimuli presented in the cortically blind hemifield might be attributed to subcortical structures such as the superior colliculus (e.g., Poppel et al. 1973), voluntary saccades are not so readily interpreted. Indeed, Troost et al. (1972) conclude that these saccades were actually generated cortically by the intact cerebral hemisphere. We will refer to such ipsilaterally directed saccades as ipsiversive saccades, and will refer to the more typical contralaterally directed saccades as contraversive saccades. We point out here that we use the terms "ipsiversive" and "contraversive" to indicate the hemisphere that putatively initiates the saccade.

Holtzman's (1984) observations on saccadic performance in callosotomy patients are also relevant to the possibility of ipsiversive saccadic control. Two patients were required to direct their gaze to a particular cell in a $2 \times 2$ matrix presented in the left visual field (LVF) based on spatial information presented in the right visual field (RVF). Based on the findings that 1) the accuracy of these saccades was greater than chance and 2) one patient was able to compare and verbally report the relative vertical alignment of the lateralized matrices, Holtzman argued that performance on this task was mediated by a weak representation of the ipsilateral visual field in each hemisphere. Further experiments suggested that only very crude spatial information may be available in this ipsilateral representation. Thus, Holtzman suggested that it is not the control of saccades which is bilateral, but the representation of visual space.

The aim of the present investigation was to determine whether the separated hemispheres in callosotomy patient 
J.W. can demonstrate bidirectional control of saccadic eye movements in response to visual information that is apparently confined to a single hemisphere. The experiment compared performance on two tasks which utilized the same stimulus conditions, but varied the response requirements. A red, green or yellow LED was presented unilaterally. In one task the subject was required to name the color. Since the right hemisphere of J.W. cannot control speech, we expected identification accuracy for LVF trials to approach chance, demonstrating the inability to transfer chromatic information to the left hemisphere speech centers. In the second task, J.W. was instructed to generate saccades either towards the light, away from it, or to refrain from responding, depending upon the color of the cue. We refer to saccades directed toward the cue as refixation saccades and those directed away from the cue as anti-saccades. ${ }^{1}$

An ability to generate anti-saccades is not readily interpreted in terms of direct collicular control since the close coupling of the sensory and motor fields in the SC is optimally designed to control foveation of eccentric targets (e.g., Wurtz and Albano 1980). Furthermore, using chromatic cues to indicate saccade direction presumably minimizes the contribution of the superior colliculus with respect to the visual processing of the cue, since coloropponent retinal ganglion cells do not project to the $\mathrm{SC}$, at least in the macaque (cf., Schiller and Malpeli 1977) and there is, at present, no evidence for color-opponent responses in the SC (e.g. Marrocco and Li 1977; Ottes, Van Gisbergen and Eggermont 1987). Thus, an ability to generate both leftward and rightward saccades based on discriminative cues that are not transferred between the hemispheres (as indicated by poor naming accuracy for LVF targets) would suggest bidirectional saccadic control by the separated cerebral hemispheres.

\section{Methods and procedure}

\section{Subjects}

Callosotomy patient, J.W., served as the subject ${ }^{2}$. He had undergone complete section of the corpus callosum 10 years previously. The completeness of this section has been confirmed using nuclear magnetic resonance scans (Gazzaniga et al. 1985). Two of the authors (HCH \& PRL) served as control observers in the saccade task.

\section{Apparatus}

Red and green signals were produced by bicolor LEDs positioned on the horizontal meridian at an eccentricity of $7.0^{\circ}$ to the right or left of

\footnotetext{
${ }^{1}$ The terms "refixation saccade" and "anti-saccade" will therefore be used simply to describe the direction of the saccade relative to the location of the target, whereas the terms "ipsiversive" and "contraversive" will be used to refer to direction of a saccade relative to the hemisphere which presumably initiates the movement

${ }^{2}$ A second callosotomy patient (VP) also participated in this experiment and produced essentially the same results. However, since VP is known to have some sparing in the splenium of her corpus callosum, we report only the results of JW
}

a white fixation light. Two yellow LEDs (also at an eccentricity of $7.0^{\circ}$ ) were used to produce the yellow cues since the yellow produced by red-green flicker of the bicolor LED was not sufficiently saturated. The yellow and bicolor LEDs were optically superimposed using beam splitters. Their relative intensities were matched using heterochromatic flicker photometry by two observers (HCH \& PRL). The matches produced by each of these observers were in close agreement, and slight differences between observers were reconciled by using average values.

Eye movements were recorded using an infrared scleral reflection device (Narco BioSystems ${ }^{\mathrm{TM}}$ Model 200). The subject viewed the stimulus display at a distance of $57 \mathrm{~cm}$. Head movements were minimized using a chin rest/head holder assembly. The experiment was controlled by a laboratory microcomputer. Eye position was digitized $(100 \mathrm{~Hz})$ from $300 \mathrm{~ms}$ before to $2200 \mathrm{~ms}$ after the presentation of the "saccade direction" cue. Eye records were analyzed off-line to determine the latency, direction and magnitude of the saccades. Saccades were detected using a velocity criterion $\left(>50^{\circ} / \mathrm{s}\right)$. Amplitude and direction were computed on the basis of calibration procedures performed prior to each experimental run in which the observer repeatedly foveated the fixation point and each of the peripheral stimuli. The performance of the data analysis algorithms was monitored by the experimenters to insure accuracy (eye blink rejection, etc.).

\section{Procedures}

J.W. participated in a color identification task and in a cued saccade task. The color identification task was used to determine whether or not the visual cues used in the saccade task could be transferred between the hemispheres. The cue was either a red, green or yellow LED and the subject was instructed to name the color as rapidly as possible. The signals were presented randomly to the right and left visual fields for $150 \mathrm{~ms}$, and eye position was monitored in order to insure that the subject's gaze was directed towards the central fixation point during each presentation of the cue.

The saccade task utilized the same stimulus conditions but required the observer to generate a saccade in a direction determined by the color of the eccentric LED. The subject was instructed to direct a saccade to the peripheral cue when it was green (refixation saccade condition), but to saccade in the opposite direction when it was red (anti-saccade condition). Yellow targets were presented on catch trials and indicated that responses should be withheld. The instructions emphasized both speed and accuracy.

The experiment was performed under conditions of low-level background illumination. Two practice sessions were given for the color naming task and three for the saccade task. J.W. participated in two experimental sessions for each task. The control observers participated in one practice and two experimental sessions for the saccade task. Each session consisted of 78 trials. The three colors were presented with equal frequency in both the left and right visual fields. The order of stimulus presentations was randomized. The color naming task preceded the saccade task. In order to minimize the possibility of cross-cueing (i.e., interhemispheric "communication" strategies that rely on "indirect" interactions between the hands and/or feet), J.W. was required to keep his hands and feet weil separated.

\section{Results}

\section{Color naming versus saccade accuracy}

J.W.'s verbal report accuracy for chromatic cues presented in the right visual field was $100 \%$. When the same cues were presented in the left visual field, naming accuracy was $34 \%$, which is chance performance. In contrast to his verbal color naming performance, J.W.'s performance on 
the saccade task for cues presented in either visual field was well above the chance level of $33.3 \%$. These data are presented in Table 1. Analysis of the errors revealed the following patterns. All false alarms which occurred in response to "no-go" cues presented to the right visual field were refixation saccades (mean latency $=477 \pm 192 \mathrm{~ms}$ ), whereas $86 \%$ of the false alarms occurring in response to "no-go" cues presented to the left visual field were antisaccades (mean latency $=535 \pm 62 \mathrm{~ms}$ ). This pattern may indicate that the false alarms were generated primarily by the left hemisphere, but other interpretations may also be viable. Errors of omission were never observed: that is, all the errors that occurred on "go" trials were direction errors (refixation saccades when anti-saccades were required and anti-saccades when refixation saccades were required).

As might be expected, normal control subjects were more accurate with respect to saccade direction $195 \%$ for refixation saccades, $94 \%$ for anti-saccades, $94 \%$ for no response trials).

\section{Saccade latency and magnitudes: Comparisons with controls}

The average saccade latencies for J.W. and the control observers are presented in Table 2. There were no instances of anticipatory saccades (i.e., $200<\mathrm{ms}$ ). The data base therefore includes all saccades occurring within the

Table 1. Proportion correct saccadic responses

\begin{tabular}{llll}
\hline \multirow{2}{*}{ Cue location } & \multicolumn{2}{l}{ Cued direction } & \\
& Left & Right & No-go \\
\hline Left visual field & 0.80 & 0.83 & 0.67 \\
Right visual field & 0.73 & 0.75 & 0.65 \\
\hline
\end{tabular}

$2.2 \mathrm{~s}$ sampling interval. Although the saccade latencies for the normal observers were shorter than J.W.'s latencies, the difference between the latencies of refixation and antisaccade were similar across the 3 observers $\left(t_{74}<1\right)$. The reasonably short latencies of J.W.'s saccades presumably minimizes the possibility that he resorted to cross-cueing strategies while performing the task. That is, the fact that J.W.'s anti-saccades were generally only about $160 \mathrm{~ms}$ slower than the control subjects appears to leave little time for the sort of "indirect communication" between the hemispheres alluded to earlier (see Methods).

The average magnitudes of both anti-saccades and refixation saccades in J.W. and the 2 control observers are also presented in Table 2. While terminal eye position of control subjects was generally more accurate with respect to the designated saccade magnitude, the pattern of J.W.'s errors was clearly not random. A representative sample of J.W.'s saccades is presented in Fig. 1. J.W. tended to undershoot the actual target location when generating contraversive saccades, but his ipsiversive saccades tended to overshoot the $7.0^{\circ}$ destination. This pattern was not apparent in the control data and we note that such overshoot of anti-saccades would have the effect of bringing the foveated target into the visual field of the ipsilateral hemisphere. Saccadic overshoot might therefore represent a strategy for distributing parafoveal information to both visual fields in the callosotomized human.

\section{Discussion}

The essential finding of this report is that peripheral cues could reliably determine the direction of the saccades in the apparent absence of interhemispheric transfer of chromatic information. The present saccade task involves an arbitrary mapping of eccentric color cues to saccade direction. The cue color must be identified before saccade
Table 2. Average latencies and magnitudes of saccades generated toward versus away from the eccentric cue

\begin{tabular}{|c|c|c|c|c|}
\hline & \multicolumn{2}{|c|}{$\begin{array}{l}J . W \\
\text { Cue location: Left visual field }\end{array}$} & \multicolumn{2}{|l|}{ Controls } \\
\hline & $\begin{array}{l}\text { Refixation } \\
\text { saccades }\end{array}$ & $\begin{array}{l}\text { Anti- } \\
\text { saccades }\end{array}$ & $\begin{array}{l}\text { Refixation } \\
\text { saccades }\end{array}$ & $\begin{array}{l}\text { Anti- } \\
\text { saccades }\end{array}$ \\
\hline \multicolumn{5}{|l|}{ Latency (ms) } \\
\hline mean & 570 & 698 & 557 & 619 \\
\hline s.d. & 137 & 355 & 88.3 & 135 \\
\hline \multicolumn{5}{|l|}{ Magnitude (deg.) } \\
\hline mean & 5.89 & 8.99 & 6.63 & 6.95 \\
\hline \multirow[t]{3}{*}{ s.d. } & 1.59 & 2.13 & 2.53 & 1.38 \\
\hline & \multicolumn{4}{|c|}{ Cue location: Right visual field } \\
\hline & $\begin{array}{l}\text { Refixation } \\
\text { saccades }\end{array}$ & $\begin{array}{l}\text { Anti- } \\
\text { saccades }\end{array}$ & $\begin{array}{l}\text { Refixation } \\
\text { saccades }\end{array}$ & $\begin{array}{l}\text { Anti- } \\
\text { saccades }\end{array}$ \\
\hline \multicolumn{5}{|l|}{ Latency (ms) } \\
\hline mean & 737 & 834 & 525 & 594 \\
\hline s.d. & 361 & 400 & 83 & 109 \\
\hline \multicolumn{5}{|l|}{ Magnitude (deg.) } \\
\hline mean & 6.4 & 8.97 & 7.3 & 4.9 \\
\hline s.d. & 2.03 & 2.75 & 1.53 & 2.17 \\
\hline
\end{tabular}


Topology of saccades : JW

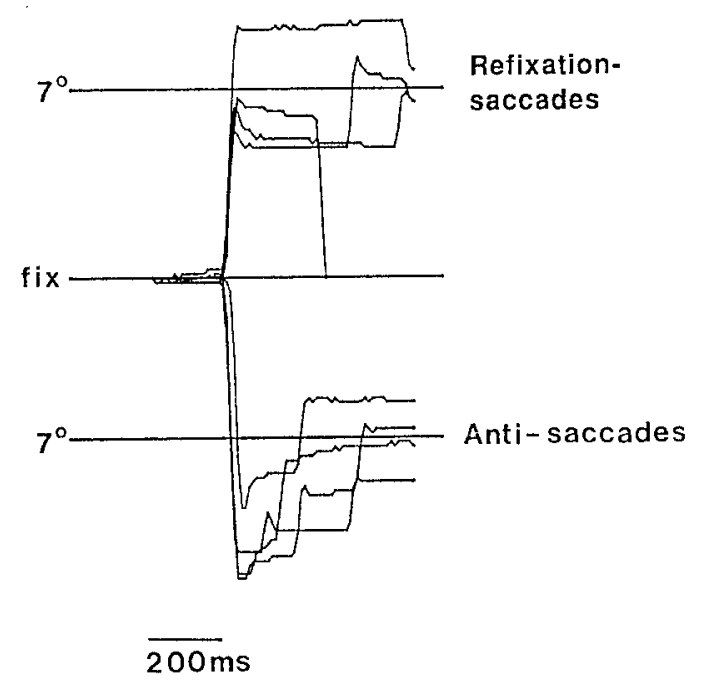

Fig. 1. Representative saccade traces from patient J.W. illustrating the tendency to overshoot the target destination in the case of ipsiversive saccades

direction can be determined. We assume therefore that performance is likely to depend on cortical processes. Given this assumption, the critical issue becomes whether saccadic responses can be attributed to the hemisphere that receives the cue or whether performance depends on some form of interhemispheric transfer.

For example, it is possible that chromatic information can transfer between the hemispheres, but in the absence of the corpus callosum, remains unavailable for verbal report. A second, related suggestion might be that a command to make a saccade can be transferred between the hemispheres. In this case, when a hemisphere receives the cue requiring an anti-saccade it signals the opposite hemisphere to generate the saccade. According to both of these accounts, all saccades are contraversive, and the present data reflect some form of extra-callosal transfer rather than ipsiversive saccadic control. The gross hypermetria evident only in J.W.'s anti-saccades suggests, however, that they are generated differently from his refixation saccades, which we presume are contralaterally controlled. Hypermetria followed by a glissade back to the target location is the type of response displayed by hemispherectomy patients. The topology of J.W.'s anti-saccades, thus, resembles that found in other cases where saccades are presumably generated by the ipsilateral cortical hemisphere (Troost et al. 1972).

We think accounts based on extra-callosal transfer are unlikely given the large body of experimental data indicating the absence of transfer in patient J.W. The absence of transfer is corroborated by a variety of direct behavioral measures including verbal report and same/different judgements, as well as more implicit measures such as priming and oculomotor performance (Gazzaniga 1987; Gazzaniga et al. 1987; Reuter-Lorenz and Baynes, in press).
Furthermore, interpretations based on transfer cannot account for the bidirectional control of voluntary saccades by hemispherectomy patients (e.g. Sharpe et al. 1979). We therefore favor interpreting the present findings in terms of the ability of a single hemisphere to generate commands for both ipsiversive and contraversive saccades.

How might a hemisphere generate saccades directed to the ipsilateral visual field? One possibility is suggested by reports that stimulation of the supplementary eye fields (SEF) can occasionally elicit ipsiversive saccades (e.g. Schlag and Schlag-Rey 1987). Secondly, there is evidence that the frontal eye fields (FEF) play an important role in generating saccades away from an eccentric target, since patients with excisions involving the FEF (with possible involvement of SEF) are particularly impaired on tasks which require saccade vectors that are opposite that required to foveate peripherally presented targets (Guitton et al. 1985). Thus, one possibility is that either the SEF or the FEF in the hemisphere contralateral to the color cue participates in generating both contraversive and ipsiversive saccades, at least in the case of patient J.W.

We recognize that this suggestion is at variance with a large body of stimulation and recording studies which indicate that the FEF generates only contraversive saccades (see Introduction). However, a review of the anatomy of the efferent projections of the FEF indicates that several established pathways might allow the FEF to execute ipsiversive saccades. First, the primate FEF projects to the contralateral as well as the ipsilateral superior colliculus ${ }^{3}$ (Distel and Fries 1982; Leichnetz et al. 1981). Second, the direct projections from the FEF to the ipsilateral paramedian pontine reticular formation (Huerta et al. 1986; Leichnetz et al. 1984) might control ipsiversive saccades, since brainstem neurons control ipsilaterally directed saccades (e.g., Fuchs et al. 1985). Another alternative might involve bilateral FEF projections to the nucleus reticularis tegmenti pontis (nRTP; Huerta et al. 1986; Leichnetz et al. 1984), which in turn project to the vermis of the cerebellum. Both nRTP and its efferent targets within the cerebellum have been shown to contain neurons which discharge in relation to saccades (Keller and Crandall 1981; Ron and Robinson 1973).

We also recognize the possibility that the putative ipsilateral control suggested here could represent a reorganization of oculomotor control which might follow commisurotomy rather than a demonstration of functional pathways that ordinarily exist in the normal brain. However, clinical examination of commissurotomy patients, including those with anterior commissural section, provides no indication of oculomotor disturbance in the acute post-operative phase (e.g. Bogen and Vogel 1962). The ability of these patients to saccade to the right or left to verbal commands, while being unable to follow verbal

\footnotetext{
${ }^{3}$ These bilateral projections emphasize the possibility that while saccades might always be generated by the contralateral superior colliculus, they could be initiated by commands from the ipsilateral cerebral hemisphere. This we would still regard as a case of ipsiversive saccadic control
} 
commands which require participation of the right hemisphere, provides anecdotal support that the left hemisphere can exert bidirectional control of saccades soon after surgery. The present investigation provides formal and quantitative evidence for a strong dissociation between standard measures of inter-hemispheric transfer of color information and the use of that same color information to control saccadic direction. While other interpretations remain possible, we suggest that some form of bidirectional control of saccade vectors represents the most parsimonius explanation of the currently available evidence.

Acknowledgements. Supported by NIH grant PO1-NS1777808. A preliminary report of these findings was presented at the annual meeting of the Society for Neuroscience, 1990. Address correspondence to $\mathrm{HCH}$ at Department of Psychology, Dartmouth College, Hanover, N.H. 03755.

\section{References}

Bizzi E, Schiller PH (1970) Single unit activity in the frontal eye fields of unanesthetized monkeys during head and eye movement. Exp Brain Res 10: 151-158

Bogen JE, Vogel PJ (1962) Cerebral commissurotomy in man. Bull. Los Angeles Neurol Soc 27: 169-172

Bruce CG, Goldberg ME (1984) Physiology of the frontal eye fields. Trends Neurosci 7: 436-441

Distel H, Fries W (1982) Contralateral cortical projections to the superior colliculus in the macaque monkey. Exp Brain Res 48: $157-163$

Fuchs AF, Kaneko CRS, Scudder CA (1985) Brainstem control of saccadic eye movements. Ann Rev Neurosci 8: 307-337

Gazzaniga MS (1987) Perceptual and attentional processes following callosal section in humans. Neuropsychologia 25: 119-133

Gazzaniga MS, Holtzman JD, Smylie CS (1987) Speech without conscious awareness. Neurology 37: 682-685

Gazzaniga MS, Holtzman JD, Deck MDF, Lee BCP (1985) NMR assessment of human callosal surgery with neuropsychological correlates. Neurology 35: 1763-1766

Guitton D, Buchtel HA, Douglas RM (1985) Frontal lobe lesions in man cause difficulties in suppressing reflexive glances and in generating goal-directed saccades. Exp Brain Res 58: 455-472

Holtzman JD (1984) Interactions between cortical and subcortical visual areas: Evidence from human commissurotomy patients. Vision Res 24: 801-813

Huerta MF, Krubitzer LA, Kaas JH (1986) Frontal eye field as defined by intracortical microstimulation in squirrel monkeys, owl monkeys and macaque monkeys: I. Subcortical connections. J Comp Neurol 253: 415-439

Keller EL, Crandall WF (1981) Neuronal activity in the nucleus reticularis tegmenti pontis in the monkey related to eye movements and visual stimulation. Ann NY Acad Sci 374: 249-261

Leichnetz GR, Smith DJ, Spencer RF (1984) Cortical projections to the paramedian tegmental and basilar pons. J Comp Neurol 228: $388-408$

Leichnetz GR, Spencer RF, Hardy SGP, Astruc J (1981) The prefrontal corticotectal projection in the monkey; an anterograde and retrograde study. Neurosci 6: 1023-1941

Marrocco RT, Li RH (1977) Monkey superior colliculus: properties of single neurons and their afferent inputs. J Neurophys 40 : $844-860$

Ottes FP, Van Gisbergen JAM, Eggermont JJ (1987) Collicular involvement in a saccadic colour discrimination task. Exp Brain Res 66: 465-478

Poppel E, Held R, Frost D (1973) Residual visual function after brain wounds involving the central visual pathways in man. Nature 243: 295-296

Reuter-Lorenz PA, Baynes K (1992) Modes of lexical access in the callosotomized brain. J Cognitive Neurosci 4: 155-164

Robinson DA (1972) Eye movements by collicular stimulation in the alert monkey. Vision Res 12: 1795-1808

Robinson DA, Fuchs AF (1969) Eye movements evoked by stimulation of the frontal eye fields. J Neurophysiol 32: 637-648

Ron S, Robinson DA (1973) Eye movements evoked by cerebellar stimulation in the alert monkey. J Neurophysiol 36: 1004-1022

Schall JD (1991a) Neuronal activity related to visually guided saccadic eye movements in the supplementary motor area of Rhesus monkeys. J Neurophysiol 66: 530-558

Schall JD (1991b) Neuronal activity related to visually guided saccadic eye movements in the frontal eye fields of Rhesus monkeys: Comparison with supplementary eye fields. J Neurophysiol 66: $559-579$

Schlag J, Schlag-Rey M (1987) Evidence for a supplementary eye field. J Neurophysiol 57: 179-200

Sharpe JA, Lo AW, Rabinovitch HE (1979) Control of the saccadic and smooth pursuit systems after cerebral hemidecortication. Brain 102: 387-403

Schiller PH, Malpeli JG (1977) Properties and tectal projections of monkey retinal ganglion cells. J Neurophysiol 40: 428-445

Sparks DL, Pollack JG (1977) The neural control of saccadic eye movements: The role of the superior colliculus. In: Bajandas FJ, Brooks BA (eds.) Eye movements. Plenum Press New York pp 179-219

Troost BT, Weber RB, Daroff RB (1972) Hemispheric control of eye movements I: Quantitative analysis of refixation saccades in a hemispherectomy patient. Arch Neurol 27: 441-448

Wurtz RH, Albano JE (1980) Visuo-motor function of the primate superior colliculus. Annu Rev Neurosci 3: 189-226 